\title{
Sistemas de transporte de drogas para o segmento posterior do olho: bases fundamentais e aplicações
}

\author{
Drug delivery systems for the posterior segment of the eye: fundamental basis \\ and applications
}

Sílvia Ligório Fialho ${ }^{1}$

Armando da Silva Cunha Júnior ${ }^{2}$

\section{RESUMO}

As doenças do segmento posterior do olho são responsáveis pela maioria dos casos de cegueira irreversível no mundo inteiro. Este cenário estimula o desenvolvimento de novas modalidades de tratamento para estas doenças. O sucesso no tratamento visa, essencialmente, o transporte de doses efetivas de drogas diretamente para os locais a serem tratados. Devido às dificuldades encontradas no transporte de drogas para o segmento posterior do olho, pesquisas têm sido realizadas no sentido de desenvolver sistemas de administração intra-oculares que permitam liberar concentrações terapêuticas das drogas por período prolongado. Tais sistemas podem proporcionar inúmeras vantagens, como: aumentar a biodisponibilidade e a concentração local da droga, atingir especificamente um tipo de tecido ou célula, reduzir a freqüência de injeções intraoculares. Tais vantagens podem aumentar o conforto do paciente e reduzir as complicações observadas com a utilização das injeções intra-oculares. Diferentes sistemas de transporte de drogas têm sido desenvolvidos com as finalidades acima descritas. Estes sistemas podem ser compostos por polímeros biodegradáveis ou não-biodegradáveis ou serem formulações lipídicas. Os sistemas de transporte de drogas são representados, principalmente, pelas micro e nanopartículas e pelos implantes, sendo eles compostos por diferentes polímeros; pelos lipossomos, que são compostos por lípides e emulsionantes; e pela iontoforese, que se baseia na aplicação de corrente elétrica. Nesta revisão, as principais características dos diferentes sistemas de transporte de drogas serão descritas, expondo suas potencialidades de aplicação clínica.

Descritores: Sistemas de liberação de medicamentos; Lipossomos; Iontoforese; Oftalmopatias/quimioterapia

\section{INTRODUCÃO}

As doenças do segmento posterior do olho são responsáveis pela maioria dos casos de cegueira irreversível no mundo inteiro. Este cenário, portanto, estimula o desenvolvimento de novas modalidades de tratamento para degenerações retinianas e doenças que atingem os tecidos posteriores do olho. O sucesso no tratamento visa, essencialmente, o transporte de doses efetivas de agentes farmacológicos diretamente para os locais a serem tratados ${ }^{(1)}$.

A baixa penetração das drogas no interior do olho limita o número de medicações indicadas para uso em oftalmologia e exige cuidado com aquelas disponíveis devido a possíveis ocorrências de efeitos adversos. A administração de drogas para o tratamento de doenças oculares deve ser 
feita, preferencialmente, por meio de vias que atinjam o tecido local, visando reduzir a ocorrência de efeitos indesejáveis e a absorção sistêmica ${ }^{(2)}$.

A administração de uma preparação pela via ocular tópica, por meio de colírios, é a mais utilizada em oftalmologia, principalmente quando a doença acomete o segmento anterior do olho. Esta via apresenta maior facilidade de aplicação e comodidade para o paciente ${ }^{(3)}$. Entretanto, as preparações tópicas são, muitas vezes, ineficazes em razão de sua baixa biodisponibilidade, devido a fatores biológicos que protegem o olho e, conseqüentemente, limitam a entrada das drogas ${ }^{(4)}$. Tais fatores incluem a impermeabilidade relativa do epitélio corneano, a dinâmica lacrimal, a drenagem nasolacrimal e a eficiente barreira hemato-ocular ${ }^{(5)}$. Estima-se que menos de 5\% da dose administrada pela via tópica é absorvida e atinge os tecidos intraoculares. Grande parte dessa dose é absorvida sistemicamente pela conjuntiva ocular e pela mucosa nasal ${ }^{(6)}$.

A via periocular é utilizada no tratamento de doenças do segmento posterior do olho por meio das injeções subconjuntival e, principalmente, da subtenoniana. A esclera constitui o tecido de entrada a esta via e algumas de suas características, tais como elevada área de superfície e fácil acesso, alto grau de hidratação, e permeabilidade que não mostra declínio apreciável com a idade, facilitam a administração de drogas por meio de injeções perioculares ${ }^{(7-10)}$. Os principais riscos relacionados a esta via incluem a perfuração do bulbo do olho, a fibrose dos músculos extra-oculares e a possível ocorrência de endoftalmite ${ }^{(11-13)}$.

As drogas administradas pela via sistêmica atingem o segmento posterior do olho através da circulação sanguínea e encontram um obstáculo anatômico importante para o tratamento de doenças oculares que é a barreira hemato-ocular, compreendida pelas barreiras hemato-aquosa e hematorretiniana. A utilização desta via na terapêutica ocular requer, geralmente, a administração de doses elevadas visando a manutenção de níveis terapêuticos intravítreos adequados por períodos prolongados, o que pode levar a sérios efeitos adversos ${ }^{(14-16)}$.

Visando a tentativa de manutenção de níveis terapêuticos adequados no segmento posterior do olho, em particular, no corpo vítreo, na retina e na coróide, a via intra-ocular tem sido a de escolha para o tratamento de diferentes doenças do olho. Utilizando esta via, pode ser evitada a ocorrência de efeitos adversos sistêmicos proporcionados por vias que não liberam a droga no local de ação ${ }^{(17)}$. No entanto, devido a uma rápida eliminação das drogas, para que se obtenha uma terapia eficaz, são necessárias múltiplas injeções intra-oculares de forma a manter a concentração do princípio ativo dentro da faixa terapêutica, no local onde se desenvolve a doença, e durante um período suficiente. As injeções intra-oculares repetidas, no entanto, podem causar sérias complicações, tais como hemorragia intraocular, descolamento de retina, endoftalmite e catarata ${ }^{(17-19)}$.

Devido a todas as dificuldades encontradas, pesquisas têm sido realizadas no sentido de desenvolver sistemas de administração intra-oculares que permitam liberar concentrações terapêuticas das drogas por um período prolongado.
Tais sistemas podem proporcionar inúmeras vantagens, como aumentar a biodisponibilidade da droga, no sentido de obter uma liberação constante e prolongada; aumentar a concentração local, sem a ocorrência de efeitos adversos sistêmicos; atingir especificamente um tipo de tecido ou célula; reduzir a freqüência de injeções intra-oculares. Tais vantagens podem aumentar o conforto do paciente e reduzir as complicações observadas com a utilização das injeções intra-oculares ${ }^{(20)}$.

Diferentes sistemas de transporte de drogas têm sido desenvolvidos com as finalidades acima descritas. Estes sistemas podem ser compostos por polímeros biodegradáveis ou não-biodegradáveis ou serem formulações lipídicas. Os sistemas de transporte de drogas são representados, principalmente, pelas micro e nanopartículas e pelos implantes, sendo eles compostos por diferentes polímeros; pelos lipossomos, que são compostos por lípides e emulsionantes; e pela iontoforese, que se baseia na aplicação de uma corrente elétrica ${ }^{(20)}$.

Nesta revisão, as principais características dos diferentes sistemas de transporte de drogas serão descritas, expondo suas potencialidades de aplicação clínica.

\section{SISTEMAS POLIMÉRICOS}

Os sistemas poliméricos de transporte de drogas são preparados a partir de diferentes polímeros, os quais podem ser biodegradáveis ou não-biodegradáveis ${ }^{(21-22)}$.

Sistemas compostos por polímeros não-biodegradáveis, principalmente, os derivados de celulose, silicones, polímeros acrílicos, polivinilpirrolidona e copolímeros dos óxidos de etileno e propileno, embora apresentem uma taxa de liberação relativamente constante, precisam, geralmente, ser removidos posteriormente, o que requer processos cirúrgicos. Já os biodegradáveis podem apresentar vantagens sobre os anteriores, pois são totalmente absorvidos pelo organismo, não necessitando remoção subseqüente ${ }^{(22)}$. A preparação de sistemas biodegradáveis requer o controle de um grande número de variáveis já que a cinética de degradação do polímero in vivo deve permanecer constante para que seja obtida uma liberação controlada da droga ${ }^{(21)}$.

Para que os sistemas poliméricos sejam eliminados do organismo, três principais mecanismos de erosão são descritos ${ }^{(23)}$ :

- difusão simples: este mecanismo está relacionado aos polímeros solúveis em água e que se tornaram insolúveis devido a uma reação química de "crosslinking". Desta forma, podese dizer que eles são insolúveis em água, mas apresentam uma matriz fortemente hidrofílica. Estes polímeros, portanto, não são adequados para a veiculação de substâncias hidrossolúveis e de baixa massa molar pois elas se difundem através da rede polimérica, independente da velocidade de degradação matricial. Entre os principais polímeros desta categoria, destacam-se a gelatina, o colágeno e o álcool polivinílico.

- erosão sem clivagem: mecanismo que ocorre com os polímeros que se tornam hidrossolúveis após reações de hidrólise ou ionização sem alterações significativas na sua massa molar. 
Por apresentarem dificuldade de eliminação, tais polímeros não podem ser empregados na forma de implantes. O Eudragit ${ }^{\circledR}$ (copolímero do éter metil vinílico e do anidrido maleico - PVM/ MA) é um exemplo de polímero que sofre este tipo de erosão.

- erosão/clivagem: neste mecanismo, os polímeros são transformados em pequenas e solúveis moléculas pela clivagem de suas cadeias. Durante este processo de degradação eles permitem a liberação da substância ativa. Este mecanismo é o que está relacionado à definição de biodegradável. Os principais polímeros que sofrem este mecanismo de erosão são os poliésteres (poli- $\varepsilon$-caprolactona, polímeros e copolímeros derivados dos ácidos lático e glicólico - PLA e PLGA), as poliamidas, os poliaminoácidos, os polialquilcianacrilatos e os poliortoésteres.

\section{Principais polímeros biodegradáveis utilizados no preparo de sistemas de transporte de drogas para o segmento posterior do olho}

Os polímeros biodegradáveis mais utilizados atualmente são os poliésteres, tais como a poli( $\varepsilon$-caprolactona), o PLA e os diferentes tipos de PLGA, sendo que os dois últimos tipos têm sido amplamente empregados ${ }^{(24)}$.

O ácido poli-lático (PLA) e os copolímeros dos ácidos lático e glicólico (PLGA) têm sido muito utilizados e estudados em sistemas de transporte prolongado de drogas para os tecidos oculares. A biodegradação desses polímeros ocorre por erosão, por meio de clivagem da cadeia polimérica por hidrólise, liberando os ácidos lático e glicólico. Esses ácidos, por serem metabólitos naturais do organismo, são eliminados pelo ciclo de Krebs na forma de gás carbônico e água ${ }^{(25)}$.

A poli(E-caprolactona) é um polímero bastante hidrofóbico e a liberação da substância ativa ocorre por meio de erosão/ clivagem da cadeia polimérica. Por apresentar uma velocidade de degradação lenta, ele pode promover a liberação de uma droga por um período de até três anos ${ }^{(23)}$. Este polímero é bastante utilizado no preparo de nanocápsulas e, atualmente, também tem sido empregado para o desenvolvimento de outros sistemas de liberação prolongada.

\section{Principais polímeros não-biodegradáveis utilizados no preparo de sistemas de transporte de drogas para o segmento posterior do olho}

Sistemas preparados com os polímeros EVA ("ethylene vinil acetate") e PVA (álcool polivinílico) foram estudados na forma de implantes. Estes polímeros atuam como reservatórios de substâncias ativas, e a liberação da droga ocorre de uma maneira mais linear do que a observada para os polímeros biodegradáveis ${ }^{(21)}$.

\section{SISTEMAS DE TRANSPORTE DE DROGAS (DRUG DELIVERY SYSTEMS)}

O primeiro sistema de liberação prolongada de drogas utilizado em oftalmologia, comercialmente disponível, foi introduzido para o tratamento de glaucoma e destinava-se à libera- ção da droga para o segmento anterior do olho ${ }^{(26)}$. No entanto, como descrito anteriormente, para o segmento posterior, o sistema de transporte deve liberar a droga na cavidade vítrea, na retina ou na coróide por um determinado período e manter a dose terapêutica no local da doença. Diferentes sistemas, tais como os implantes sólidos, as micro e nanopartículas, os lipossomos e a iontoforese, têm sido estudados como potenciais no transporte prolongado de drogas ${ }^{(27)}$.

\section{Implantes sólidos}

Implantes preparados a partir de sistemas poliméricos podem ser aplicados em diferentes regiões do olho. Ordenando-se as regiões da mais superficial à mais profunda, temos: região subconjuntival, região subtenoniana, esclera e o interior do bulbo do olho (câmara anterior e corpo vítreo). Em geral quanto mais profunda a região, mais delicado o procedimento e mais eficaz a concentração da droga no vítreo e na retina ${ }^{(22,26)}$.

Os implantes intravítreos evitam o problema de redução da transparência do meio ocular, observado com a utilização de outros tipos de sistemas aplicados na forma de dispersão, e têm sido investigados para o tratamento de diferentes doenças intra-oculares e também para aplicação em cirurgias de catarata $^{(28-37)}$.

Implantes não-biodegradáveis de liberação lenta foram aprovados para uso clínico nos Estados Unidos da América (EUA): o Ocusert ${ }^{\circledR}$ (Alza, EUA), um dispositivo conjuntival que libera pilocarpina, o Vitrasert ${ }^{\circledR}$ (Bausch \& Lomb, EUA), um implante intravítreo contendo ganciclovir que tem sido usado em pacientes com a síndrome da imunodeficiência adquirida (SIDA) para o tratamento de retinite por citomegalovírus (CMVR) e o Retisert ${ }^{\circledR}$ (Bausch \& Lomb, EUA), um implante intravítreo contendo fluocinolona indicado para o tratamento de uveíte não infecciosa crônica ${ }^{(38)}$. O problema destes implantes é que, como os polímeros não são biodegradáveis, é necessária a remoção do sistema após completa liberação da droga ou, caso não sejam removidos, eles permanecem no local, até que sejam retirados por meio de processos cirúrgicos.

Implantes intra-oculares preparados com polímeros biodegradáveis não precisam ser posteriormente removidos, pois são completamente metabolizados e eliminados pelo organismo. O desenvolvimento destes sistemas pode se tornar um grande avanço já que eles poderão ser capazes de manter concentrações da droga no local, dentro da faixa terapêutica e por longo período ${ }^{(29,34,37,39-40)}$. Um sistema de liberação de dexametasona para o tratamento de inflamação após cirurgia de catarata, o Surodex ${ }^{\circledR}$ (Oculex Pharmaceuticals, Inc, EUA) foi avaliado clinicamente ${ }^{(37,41)}$. Este implante foi preparado com PLGA e continha $60 \mu \mathrm{g}$ da droga. O sistema foi capaz de promover liberação da droga por um período de, aproximadamente, 7 a 10 dias. Os estudos clínicos revelaram, ainda, que o implante foi bem tolerado e efetivo contra a inflamação. Este sistema já foi aprovado para utilização clínica na Singapura e no México e completou os estudos clínicos de fase III, mas ainda aguarda a aprovação do Food and Drug Administration (FDA) para utilização nos EUA ${ }^{(42)}$. 
Um outro implante em avaliação, o Posurdex ${ }^{\circledR}$ (Allergan, USA), é capaz de liberar a dexametasona no segmento posterior do olho. Este sistema encontra-se em estudos clínicos de fase III para o tratamento de edema macular associado ao diabetes e outras doenças (Allergan, Inc., 2005). A empresa aguarda a aprovação do FDA para comercialização do produto provavelmente para o ano de $2007^{(43)}$.

Em nosso laboratório, e em colaboração com oftalmologistas da Faculdade de Medicina de Ribeirão Preto da Universidade de São Paulo, um implante biodegradável intravítreo contendo dexametasona foi desenvolvido(44) (Figura 1). Os estudos pré-clínicos mostraram uma liberação da droga, dentro da faixa terapêutica, por um período de 8 semanas, podendo ainda ser prolongado por maior tempo. Os resultados deste trabalho mostraram, ainda, uma ausência de alteração nas estruturas oculares e a anatomia normal da retina foi preservada durante todo o período de avaliação.

\section{Micro e nanopartículas}

As micro e nanopartículas são transportadores coloidais de tamanho micro ou nanométrico, respectivamente. Elas podem ser preparadas a partir de polímeros sintéticos, tais como o PLA/PLGA e a poli(E-caprolactona) ${ }^{(45)}$.

A vantagem de utilização destes sistemas é que, por se apresentarem na forma de dispersão coloidal, a administração pode ser realizada por meio de injeções, evitando complicações dos processos cirúrgicos encontrados na aplicação de implantes intravítreos ${ }^{(20)}$.

As micro e nanopartículas são estruturas poliméricas contendo a droga que são, frequentemente, classificados pelo tamanho, sendo que as micropartículas geralmente apresentam tamanho superior a $1 \mu \mathrm{m}$ e as nanopartículas tamanho inferior a $1 \mu \mathrm{m}$. Dependendo da estrutura estes sistemas podem, ainda, ser de dois tipos: matriciais (ou monolíticos), no caso das nano e microesferas, e reservatórios, no caso das nano e microcápsulas ${ }^{(21-22)}$ (Figura 2). No sistema matricial, a droga se encontra dispersa na matriz polimérica. A liberação da droga, caso se trate de um sistema biodegradável, ocorre por difusão pelos poros da matriz, por degradação do polímero ou por uma combinação dos dois mecanismos. Quando se utilizam polímeros não-biodegradáveis, a liberação ocorre apenas por um processo de difusão lenta pela matriz. No sistema do tipo reservatório, preparados com polímeros biodegradáveis ou não-biodegradáveis, a droga se encontra em uma cavidade central envolta por uma membrana polimérica, a qual controla sua taxa de liberação ${ }^{(21-22)}$.

O objetivo no desenvolvimento de micro e nanopartículas tem sido a obtenção de formulações injetáveis de longa ação e com características de atuação em tecidos ou células específicos. Estes sistemas estão sendo estudados para o transporte ocular de drogas há 15 anos ${ }^{(45)}$.

Os polímeros biodegradáveis que têm sido estudados para o desenvolvimento de micropartículas para o transporte de drogas são a gelatina, a albumina, os poliortoésteres, os po- lianidridos, e os poliésteres, principalmente os polímeros derivados dos ácidos lático e glicólico [ácido poli-lático (PLA), ácido poli-glicólico (PGA) e ácido poli-lático-co-glicólico (PLGA)]. Os poliésteres acima descritos têm sido os mais freqüentemente empregados no desenvolvimento de microesferas para aplicação intravítrea ${ }^{(45)}$.

Entre as partículas poliméricas potencialmente aplicáveis para administração intra-ocular, as microesferas são as mais comumente utilizadas $^{(45)}$ (Figura 3).

Estudos da aplicação intravítrea de microesferas investigaram a liberação do ganciclovir ${ }^{(46-47)}$, do ácido retinóico ${ }^{(48)}$, do 5 -fluorouracila ${ }^{(49)}$ e da fluoresceína ${ }^{(50)}$. Os resultados destes trabalhos mostraram que, após injeção das microesferas na

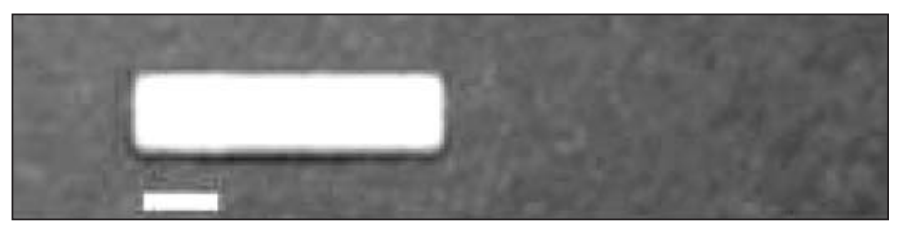

Figura 1 - Fotografia de um implante biodegradável intravítreo contendo dexametasona (Barra= $1 \mathrm{~mm}$ )

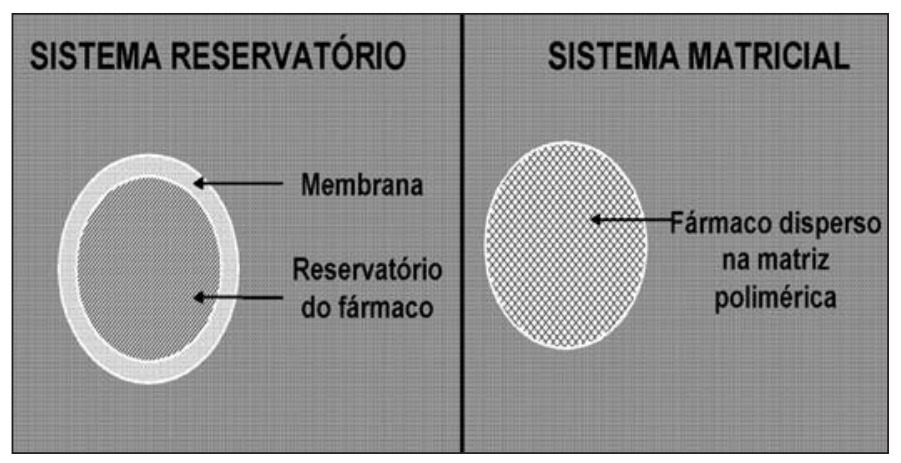

Figura 2 - Representação esquemática da estrutura de um sistema reservatório e matricial de micro e nanopartículas

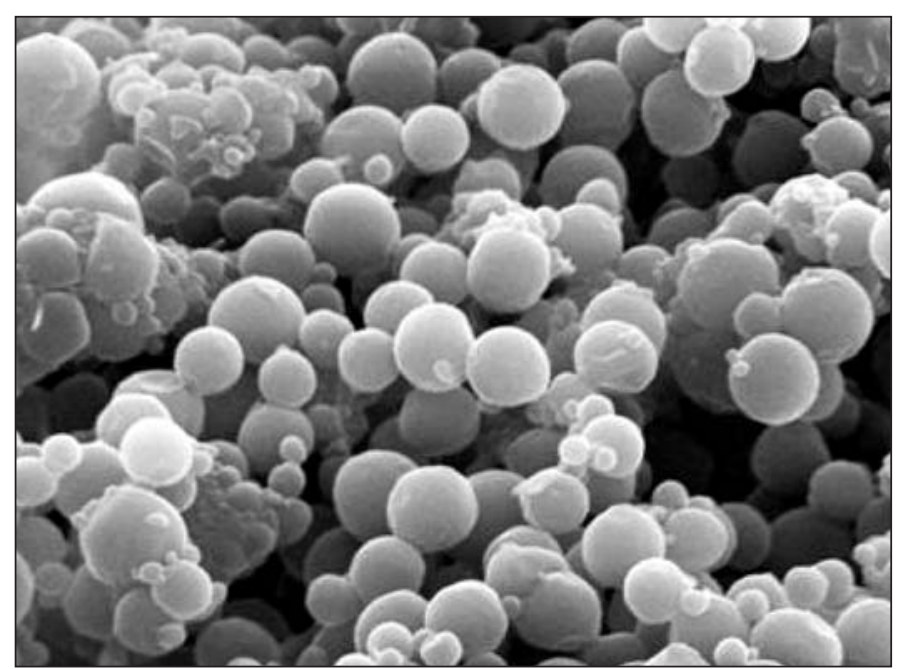

Figura 3 - Fotomicrografia eletrônica de varredura de microsferas 
cavidade vítrea, houve uma liberação das substâncias durante um período de duas a oito semanas.

As micropartículas, como as microesferas, apresentam como vantagem a administração por meio de injeções intravítreas. Estudos mostraram que é possível a liberação de certas drogas por longos períodos e que estes sistemas apresentam biocompatibilidade com os tecidos oculares. As limitações da utilização das microesferas estão relacionadas a sua potencialidade em causar deficiência visual pois a presença das partículas suspensas na cavidade vítrea podem provocar embaçamento da visão ${ }^{(51)}$. Além disso, as microesferas, por apresentarem baixa quantidade de droga encapsulada, podem requerer a administração repetida das injeções intra-oculares em intervalos semanais ou mensais, o que pode causar desconforto para o paciente e potenciais complicações ${ }^{(45)}$.

\section{Lipossomos}

Os lipossomos são sistemas de transporte de drogas coloidais que se formam espontaneamente quando certos lípides são hidratados em meio aquoso ${ }^{(52)}$.

Os lipossomos são compostos por substâncias relativamente biocompatíveis e biodegradáveis e consistem de uma ou mais bicamadas lipídicas que circundam um meio aquoso. Os lipossomos são constituídos por lípides naturais e/ou sintéticos, tais como os fosfolípides e os esfingolípides, e podem apresentar, também, outros componentes na bicamada, como colesterol e lípides conjugados com polímeros hidrofílicos ${ }^{(52)}$ (Figura 4). Os produtos ativos podem ser incorporados tanto na solução aquosa como nas próprias bicamadas de fosfolípides, permitindo, dessa forma, o transporte de agentes hidrofílicos e hidrofóbicos em uma mesma formulação ${ }^{(20)}$. Os lipossomos podem ser carregados positivamente, negativamente ou serem neutros, dependendo da sua composição.

O tamanho dos lipossomos pode variar de vesículas muito pequenas $(0,025 \mu \mathrm{m})$ a grandes $(2,5 \mu \mathrm{m})$, apresentando uma única ou múltiplas bicamadas. Com relação ao tamanho e o

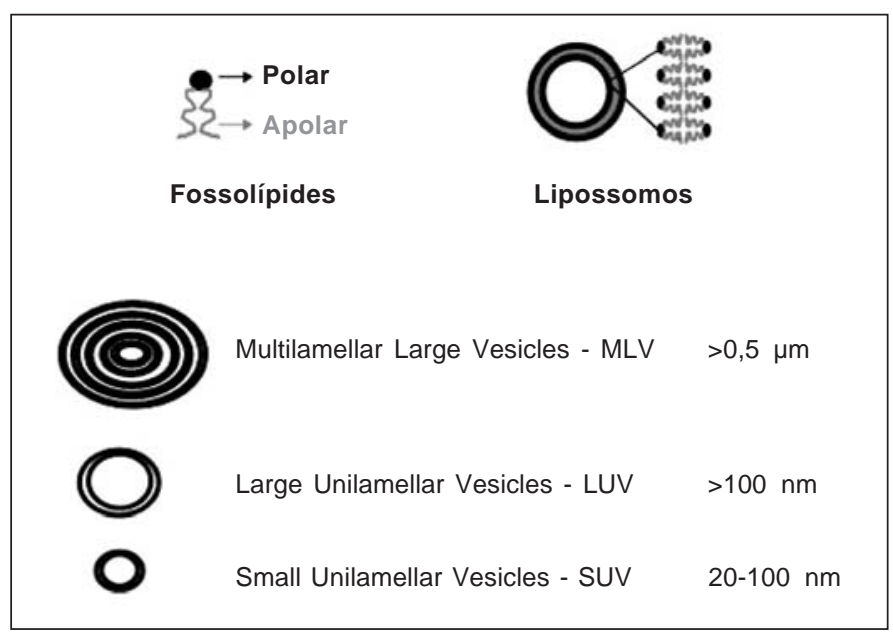

Figura 4 - Representação esquemática dos fosfolípides, da estrutura e dos tipos de lipossomos número de bicamadas, os lipossomos podem ser classificados em vesículas multilamelares (Multilamellar large vesicles MLV); vesículas unilamelares grandes (Large unilamellar vesicles - LUV) e vesículas unilamelares pequenas (Small unilamellar vesicles - SUV) ${ }^{(52)}$ (Figura 4).

A farmacocinética e a biodistribuição in vivo destes sistemas pode ser controlada pelo tamanho e pela composição lipídica dos lipossomos. O tamanho da vesícula é um parâmetro na determinação da penetração e da meia-vida destes sistemas, e tanto o tamanho quanto o número das bicamadas influenciam a taxa de encapsulamento da droga a ser veiculada ${ }^{(20)}$.

Um exemplo de formulação lipossomal encontrada no mercado para o tratamento de algumas doenças intra-oculares, e que é bastante utilizada atualmente, é o Visudyne ${ }^{\circledR}$ (Novartis Pharmaceuticals Corporation, EUA) que apresenta como princípio ativo a verteporfina ${ }^{(53-54)}$. Esta formulação, aplicada pela via intravenosa, é indicada, principalmente, para o tratamento de neovascularização em pacientes com degeneração macular ${ }^{(53)}$.

As limitações da utilização dos lipossomos estão relacionadas, principalmente, ao embaçamento da visão causado pela presença das partículas dos lipossomos suspensas na cavidade vítrea, e à dificuldade de armazenamento devido à baixa estabilidade que apresentam. Além disso, os métodos de preparo são relativamente complexos quando comparado aos utilizados para o preparo de implantes sólidos e de microesferas ${ }^{(51)}$.

\section{Iontoforese}

A iontoforese, termo grego que significa transferência iônica, é um procedimento em que íons são conduzidos em um tecido pela ação de uma corrente elétrica de baixa intensidade, a qual modifica a permeabilidade das células e facilita a penetração de drogas ionizadas através das membranas biológicas ${ }^{(55-56)}$.

A iontoforese é definida como um processo de transferência de drogas ionizadas através de uma membrana, como a córnea, para um outro tecido pelo uso de uma diferença de potencial elétrico entre dois eletrodos. O transporte por iontoforese depende de uma força eletromotiva que repele íons de um eletrodo de mesma carga e os faz migrar para um eletrodo de carga oposta ${ }^{(55-56)}$.

Esta técnica apresenta um interesse particular no transporte de várias drogas, para diferentes tecidos do olho, incluindo o segmento posterior e, principalmente, para o espaço subretiniano para atingir a retina e a coróide ${ }^{(55,57)}$. É uma técnica não-invasiva e sua aplicação pode ser repetida quantas vezes forem necessárias, com baixos riscos de efeitos adversos. Por meio dela é possível transportar moléculas clássicas, novas drogas, assim como oligonucleotídeos ${ }^{(55,58)}$. A iontoforese permite novas modalidades terapêuticas para tratar doenças oculares degenerativas, já que ela contribui para o acúmulo preferencial de drogas na úvea e na retina, tecidos alvos para possível terapia gênica de doenças oculares humanas.

As maiores desvantagens do transporte de drogas por iontoforese são os riscos de queimaduras e choques resultantes da utilização de correntes elétricas elevadas e por longos 
períodos. Os riscos de queimadura podem ser causados por fatores como o contato dos eletrodos com o olho, superdosagem, presença de lesões no local de aplicação, alterações significativas de $\mathrm{pH}$ e elevada intensidade da corrente ${ }^{(55-56)}$.

Por meio da iontoforese é possível potencializar a administração intra-ocular e atingir os tecidos alvos. Mas, esta técnica não é um sistema de liberação prolongada e não dispensa a necessidade de repetição do tratamento ${ }^{(59)}$.

Alguns sistemas encontram-se, atualmente, em diferentes fases de estudos clínicos nos Estados Unidos e Europa, tais como o Eyegate ${ }^{\circledR}$ (Eyegate Pharma, França) ${ }^{(60)}$, que está sendo testado para o transporte de drogas visando o tratamento de retinopatia diabética, degeneração macular e retinite pigmentosa; o Ocuphor ${ }^{\circledR}$ (Iomed Inc., EUA ${ }^{(61)}$ em estudo para o tratamento de inflamações oculares por meio do transporte de diclofenaco de sódio; e o Visulex (Aciont Inc., EUA), que está sendo testado para o tratamento de uveíte posterior empregando-se, como droga, o fosfato sódico de dexametasona ${ }^{(62)}$.

\section{CONCLUSÃO}

Novos sistemas de transporte de drogas para o segmento posterior do olho, tais como os implantes sólidos, as micro e nano-partículas, os lipossomos e a iontoforese, apresentam um grande potencial para o tratamento de doenças vitreorretinianas.

Os sistemas injetáveis apresentam a vantagem de não serem necessários procedimentos cirúrgicos, mas os implantes sólidos, apesar de requererem cirurgia para implantação, apresentam uma cinética de liberação mais prolongada.

Apesar das intensas pesquisas, é quase certo que nenhum sistema permitirá o transporte de todos os agentes terapêuticos existentes e nem poderão ser aplicados para todas as doenças. A verdadeira inovação encontra-se no desenvolvimento de procedimentos complementares e provavelmente, uma combinação entre diferentes sistemas, o que poderá permitir uma maior eficiência no tratamento de doenças que acometem o segmento posterior do olho.

\section{ABSTRACT}

The diseases of the posterior segment of the eye are responsible for most cases of irreversible blindness worldwide. These conditions stimulate the development of new modalities of treatment for vitreoretinal diseases. The success in the treatment aims, mainly, the delivery of effective doses of pharmacological agents directly to the target sites. Because of the difficulties in delivering drugs to the posterior segment of the eye, the development of intraocular delivery systems that allow the delivery of therapeutic concentrations of drugs for long periods are being studied. These systems offer many advantages, such as increase in drug bioavailability, obtaining constant and sustained drug release, to achievement of elevated local concentrations of drugs without systemic side effects, targeting one specific tissue or cell type, reducing the frequency of intraocular injections. These advantages can increase the comfort of the patient and reduce the complications observed with intraocular injections. Several drug delivery systems are being developed with the above described purposes. These systems may be prepared with biodegradable or non-biodegradable polymers or they may be lipid formulations. The drug delivery systems are represented, mainly, by micro- e nanoparticles and implants, composed of different polymers; by liposomes, which are made of lipids and surfactants; and by iontophoresis, that is based on the application of an electric current. In this review, the main characteristics of the different drug delivery systems will be shown, with their potentialities of clinical application.

Keywords: Drug delivery systems; Liposomes; Iontophoresis; Eye diseases/drug therapy

\section{REFERÊNCIAS}

1. Geroski DH, Edelhauser HF. Transscleral drug delivery for posterior segment disease. Adv Drug Deliv Rev. 2001;52(1):37-48.

2. Ogura Y. Drug delivery to the posterior segments of the eye. Adv Drug Deliv Rev. 2001;52(1):1-3.

3. Urtti A, Salminen L. Minimizing systemic absorption of topically administered ophthalmic drugs. Surv Ophthalmol. 1993;37(6):435-56.

4. Davies NM. Biopharmaceutical considerations in topical ocular drug delivery. Clin Exp Pharmacol Physiol. 2000;27(7):558-62.

5. Ding S. Recent developments in ophthalmic drug delivery. Pharm Sci Technol Today. 1998;1(8):328-35.

6. Järvinen K, Järvinen T, Urtti A. Ocular absorption following topical delivery. Adv Drug Deliv Rev. 1995;16(1):3-19.

7. Foster CS, Maza MS. The sclera. New York: Springer-Verlag; c1994.

8. Olsen TW, Edelhauser HF, Lim JI, Geroski DH. Human scleral permeability. Effects of age, cryotherapy, transscleral diode laser, and surgical thinning. Invest Ophthalmol Vis Sci. 1995;36(9):1893-903.

9. Olsen TW, Aaberg SY, Geroski DH, Edelhauser HF. Human sclera: thickness and surface area. Am J Ophthalmol. 1998;125(2):237-41.

10. Ambati J, Canakis CS, Miller JW, Gragoudas ES, Edwards A, Weissgold DJ, et al. Diffusion of high molecular weight compounds through sclera. Invest Ophthalmol Vis Sci. 2000;41(5):1181-5.

11. Hida T, Chandler D, Arena JE, Machemer R. Experimental and clinical observations of the intraocular toxicity of commercial corticosteroid preparations. Am J Ophthalmol. 1986;101(2):190-5.

12. Smith JR, George RK, Rosenbaum JT. Lower eyelid herniation of orbital fat may complicate periocular corticosteroid injection. Am J Ophthalmol. 2002; 133(6):845-7.

13. Gupta OP, Boynton JR, Sabini P, Markowitch W Jr, Quatela VC. Proptosis after retrobulbar corticosteroid injections. Ophthalmology. 2003;110(2):443-7.

14. Foulds WS, Moseley H, Eadie A, McNaught E. Vitreal, retinal, and pigment epithelial contribution to the posterior blood-ocular barrier. Trans Ophthalmol Soc U K. 1980;100(3):341-2.

15. Cunha-Vaz JG. The blood-ocular barriers: past, present, and future. Doc Ophthalmol. 1997;93(1-2):149-57.

16. Cunha-Vaz JG. The blood-retinal barriers system. Basic concepts and clinical evaluation. Exp Eye Res. 2004;78(3):715-21.

17. Peyman GA, Ganiban GJ. Delivery systems for intraocular routes. Adv Drug Deliv Rev. 1995;16(1):107-23.

18. Velez G, Whitcup SM. New developments in sustained release drug delivery for the treatment of intraocular disease. Br J Ophthalmol. 1999;83(11):1225-9.

19. Yasukawa T, Ogura Y, Tabata Y, Kimura H, Wiedemann P, Honda Y. Drug delivery systems for vitreoretinal diseases. Prog Retin Eye Res. 2004;23(3):253-81.

20. Behar-Cohen F. Systèmes de délivrance des médicaments pour le segment antérieur: bases fondamentales et applications cliniques. J Fr Ophtalmol. 2002;25(5):537-44. 
21. Dash AK, Cudworth GC $2^{\text {nd }}$. Therapeutic applications of implantable drug delivery systems. J Pharmacol Toxicol Methods. 1998;40(1):1-12.

22. Kimura H, Ogura Y. Biodegradable polymers for ocular drug delivery. Ophthalmologica. 2001;215(3):143-55.

23. Merkli A, Tabatabay C, Gurny R, Heller J. Biodegradable polymers for the controlled release of ocular drugs. Prog Polym Sci. 1998;23(3):563-80.

24. Jain R, Shah NH, Malick AW, Rhodes CT. Controlled drug delivery by biodegradable poly(ester) devices: different preparative approaches. Drug Dev Ind Pharm. 1998;24(8):703-27.

25. Saettone MF, Salminen L. Ocular inserts for topical delivery. Adv Drug Deliv Rev. 1995;16:95-106.

26. Athanasiou KA, Niederauer GG, Agrawal CM. Sterilization, toxicity, biocompatibility and clinical applications of polylactic acid/polyglycolic acid copolymers. Biomaterials. 1996;17(2):93-102.

27. Fialho SL, Rego MGB, Cardillo JA, Siqueira RC, Jorge R, Cunha Júnior AS. Implantes biodegradáveis destinados à administração intra-ocular. Arq Bras Oftalmol. 2003;66(6):891-6.

28. Sanborn GE, Anand R, Torti RE, Nightingale SD, Cal SX, Yates B, et al. Sustained-release ganciclovir therapy for treatment of cytomegalovirus retinitis. Use of an intravitreal device. Arch Ophthalmol. 1992;110(2):188-95.

29. Hashizoe M, Ogura Y, Kimura H, Moritera T, Honda Y, Kyo M, et al. Scleral plug of biodegradable polymers for controlled drug release in the vitreous. Arch Ophthalmol. 1994;112(10):1380-4.

30. Rubsamen PE, Davis PA, Hernandez E, O'Grady GE, Cousins SW. Prevention of experimental proliferative vitreoretinopathy with a biodegradable intravitreal implant for the sustained release of fluorouracil. Arch Ophthalmol. 1994;112(3): 407-13.

31. Cheng CK, Berger AS, Pearson PA, Ashton P, Jaffe GJ. Intravitreal sustained-release dexamethasone device in the treatment of experimental uveitis. Invest Ophthalmol Vis Sci. 1995;36(2):442-53.

32. Kunou N, Ogura Y, Hashizoe M, Honda Y, Hyon SH, Ikada Y. Controlled intraocular delivery of ganciclovir with use of biodegradable scleral implant in rabbits. J Control Release. 1995;37(1-2):143-50.

33. Yang CS, Khawly JA, Hainsworth DP, Chen SN, Ashton P, Guo H, Jaffe GJ. An intravitreal sustained-release triamcinolone and 5-fluorouracil codrug in the treatment of experimental proliferative vitreoretinopathy. Arch Ophthalmol. 1998;116(1):69-77.

34. Zhou T, Lewis H, Foster RE, Schwendeman SP. Development of a multipledrug delivery implant for intraocular management of proliferative vitreoretinopathy. J Control Release. 1998;55(2-3):281-95.

35. Velez G, Whitcup SM. New developments in sustained release drug delivery for the treatment of intraocular disease. Br J Ophthalmol. 1999;83(11):1225-9.

36. Jaffe GJ, Ben-Nun J, Guo H, Dunn JP, Ashton P. Fluocinolone acetonide sustained drug delivery device to treat severe uveitis. Ophthalmology. 2000; 107(11):2024-33.

37. Tan DT, Chee SP, Lim L, Theng J, Van Ede M. Randomized clinical trial of Surodex steroid drug delivery system for cataract surgery: anterior versus posterior placement of two Surodex in the eye. Ophthalmology. 2001;108 (12):2172-81

38. Food and Drug Administration. FDA news. Product approvals. [cited 2005 Ago 8]. Available from: < http://www.fda.gov/opacom/7approvl.html>.

39. Kimura H, Ogura Y, Hashizoe M, Nishiwaki H, Honda Y, Ikada Y. A new vitreal drug delivery system using an implantable biodegradable polymeric device. Invest Ophthalmol Vis Sci. 1994;35(6):2815-9.

40. Sakurai E, Nozaki M, Okabe K, Kunou N, Kimura H, Ogura Y. Scleral plug of biodegradable polymers containing tacrolimus (FK506) for experimental uveitis. Invest Ophthalmol Vis Sci. 2003;44(11):4845-52.

41. Tan DT, Chee SP, Lim L, Lim AS. Randomized clinical trial of a new dexamethasone delivery system (Surodex) for treatment of post-cataract surgery inflammation. Ophthalmology. 1999;106(2):223-31.

42. The Wall Street Transcript. Dain rauscher wessels healthcare \& life sciences special. CEO/COMPANY Interview. In: Donald J. Eaton. Oculex Pharmaceuticals, Inc; December 2000. [cited 2005 Aug 8]. Available from: http://www. twst.com/pdf/oculex.pdf\#search='surodex\%20approval'

43. Allergan Completes Acquisition of Oculex Pharmaceuticals, Inc. [text on the Internet]. USA: Allergan Inc; c2006. [cited 2006 Feb 1]. Available from:http:// www.allergan.com/site/investors/pr_details.asp?id=123213.

44. Fialho SL, da Silva Cunha A. Manufacturing techniques of biodegradable implants intended for intraocular application. Drug Deliv. 2005;12(2):109-16.

45. Herrero-Vanrell R, Refojo MF. Biodegradable microspheres for vitreoretinal drug delivery. Adv Drug Deliv Rev. 2001;52(1):5-16.

46. Refojo MF, Herrero-Vanrell R. Sustained delivery of ganciclovir from biodegradable poly (DL-lactide-co-glycolide) (PLGA) microspheres [abstract]. Invest Ophthalmol Vis Sci. 1996;37:18S

47. Herrero-Vanrell R, Barcia E, Negro S, Refojo MF. Development of ganciclovir microspheres from poly(DL-lactide-co-glycolide) acid for the treatment of AIDSrelated cytomegalovirus retinitis. STP Pharma Sciences. 1998;8237-40S.

48. Giordano GG, Refojo MF, Arroyo MH. Sustained delivery of retinoic acid from microspheres of biodegradable polymer in PVR. Invest Ophthalmol Vis Sci. 1993;34(9):2743-51.

49. Moritera T, Ogura Y, Honda Y, Wada R, Hyon SH, Ikada Y. Microspheres of biodegradable polymers as a drug-delivery system in the vitreous. Invest Ophthalmol Vis Sci. 1991;32(6):1785-90.

50. Khoobehi B, Stradtmann MO, Peyman GA, Aly OM. Clearance of sodium fluorescein incorporated into microspheres from the vitreous after intravitreal injection. Ophthalmic Surg. 1991;22(3):175-80.

51. Colthurst MJ, Williams RL, Hiscott PS, Grierson I. Biomaterials used in the posterior segment of the eye. Biomaterials. 2000;21(7):649-65.

52. Sharma A, Sharma US. Liposomes in drug delivery: progress and limitations. Int J Pharm. 1997;154:123-40.

53. Full prescribing Information for Visudyne [text on the Internet]. St. Louis, MO NOVARTIS Pharmaceuticals Corporation; c2006. [cited 2005 Jul 4]. Available from: http://www.pharma.us.novartis.com/product/pi/pdf/visudyne.pdf.

54. Ichikawa K, Takeuchi Y, Yonezawa S, Hikita T, Kurohane K, Namba Y, Oku N. Antiangiogenic photodynamic therapy (PDT) using Visudyne causes effective suppression of tumor growth. Cancer Lett. 2004;205(1):39-48.

55. Fialho SL, Cunha Júnior AS. Iontoforese no transporte ocular de drogas. Arq Bras Oftalmol. 2004;67(5):839-45.

56. Kalia YN, Naik A, Garrison J, Guy RH. Iontophoretic drug delivery. Adv Drug Deliv Rev. 2004;56(5):619-58. Review.

57. Lai PM, Roberts MS. Iontophoresis. In: Roberts MS. Dermal absorption and toxicity assessment New York: Marcel Dekker; 1998. p.371-414. [Drugs and the pharmaceutical sciences: a series of textbooks and monographs].

58. Sarraf D, Lee DA. The role of iontophoresis in ocular drug delivery. J Ocul Pharmacol. 1994;10(1):69-81.

59. Behar-Cohen F, El Aouni A, Le Rouic JF, Parel JM, Renard G, Chauvaud D. [Iontophoresis: past and future]. J Fr Ophtalmol. 2001;24(3):319-27. French.

60. Technology: Eyegate [text on the Internet]. Paris: EyeGate Pharma; c2005. [cited 2006 Feb 10]. Available form: http://www.eyegatepharma.com/webpages/ eyegate.htm

61. Fischer GA, Parkinson TM, Szlek MA. OcuPhor ${ }^{\mathrm{TM}}$ - The future of ocular drug delivery [text on the Internet]. [cited 2006 Feb 10]. Available from: http://www.drugdeliverytech.com/cgi-bin/articles.cgi?idArticle=62. Drug delivery technology. Looking into iontophoresis [text on the Internet]. [cited 2006 Feb 10]. Available from: http://www.aciont.com/DrugDelivery Technology042004.pdf.

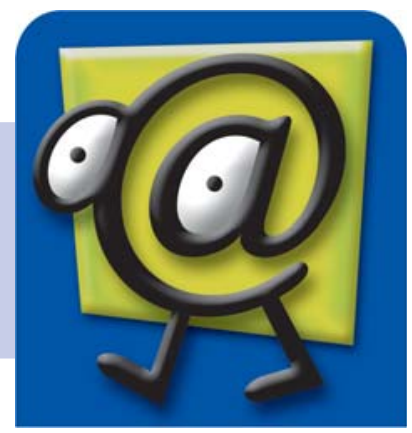

